\section{Síncope secundario a mixoma gigante del anillo mitral. Reporte de un caso}

\author{
JULIÁN VEGA ${ }^{1}$, LUIGI GABRIELLI' ${ }^{1}$, GABRIEL OLIVARES ${ }^{2}$, \\ SAMUEL CÓRDOVA ${ }^{1}$, MANUEL MÉNDEZ ${ }^{1}$, RODRIGO GONZÁLEZ ${ }^{2}$
}

\section{Large mitral annulus myxoma presenting with syncope. Report of one case}

We report a 23-year-old woman, with three recent exertional syncopes. Transthoracic (TTE) and transesophageal (TEE) echocardiography found a large heterogeneous mass (38 $\times 35 \mathrm{~mm}$ ) arising from the posterior mitral annulus, protruding in systole through the left ventricular outflow tract (LVOT). Heart MRI confirmed the echocardiography findings, suggesting a cardiac myxoma. Cardiac surgery accomplished the complete resection of the lesion, confirming a mass arising from the posterior mitral annulus and preserving mitral anatomy and function. Pathology was positive for a myxoma. Uneventful evolution allowed the discharge of the patient at the fifth postoperative day. Control TTE discarded any complication.

(Rev Med Chile 2016; 144: 1617-1620)

Key words: Heart Neoplasms; Myxoma; Mitral Valve Annuloplasty; Syncope.

\author{
'Laboratorio de Ecocardiografía, \\ División de Enfermedades \\ Cardiovasculares. Facultad de \\ Medicina. Pontificia Universidad \\ Católica de Chile. Santiago, Chile. \\ ${ }^{2}$ Sección de Cirugía Cardíaca, \\ División de Enfermedades \\ Cardiovasculares. Facultad de \\ Medicina. Pontificia Universidad \\ Católica de Chile. Santiago, Chile. \\ Sin apoyo financiero. \\ Recibido el 2 de marzo de 2016, \\ aceptado el 11 de octubre de \\ 2016 \\ Correspondencia a: \\ Dr. Julián Vega Adauy \\ División de Enfermedades \\ Cardiovasculares, Laboratorio de \\ Ecocardiografía, Hospital Cínico \\ Pontificia Universidad Católica \\ de Chile. \\ Marcoleta 367, Santiago, Chile. \\ julianvega@gmail.com
}

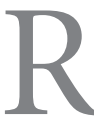
eportamos el caso de una mujer de 23 años, con historia de tres eventos sincopales relacionados con esfuerzo. En el examen físico se pesquisó un soplo sistólico, paraesternal izquierdo, grado V/VI, siendo referida a nuestro laboratorio para una ecocardiografía. Se realizó ecocardiografía transtorácica (ETT) y transesofágica (ETE) que mostraron el ventrículo izquierdo de tamaño y función normal, con una masa heterogénea multilobulada de dimensiones aproximadas $38 \times 35 \mathrm{~mm}$ (Figura 1), que se encontraba adherida al anillo posterior mitral, hacia los segmentos $\mathrm{P} 2$ y P3, a través de un pedículo de $20 \mathrm{~mm}$ (Figura 2). Dicha masa protruía en sístole hacia el tracto de salida del ventrículo izquierdo (TSVI), generando gradientes máximos de 30 $\mathrm{mmHg}$, que no se modificaban tras maniobra de Valsalva. Además, se objetivó una insuficiencia mitral leve protosistólica. Se amplió el estudio con una resonancia magnética (RM) de corazón, que informó una masa de partes blandas de 45 $\mathrm{mm} \times 24 \mathrm{~mm}$, con su base de implantación bajo el plano del velo posterior mitral hacia su segmento P3. Las secuencias de caracterización tisular y post gadolinio sugirieron un mixoma cardíaco. Se realizó la resección tumoral, mediante un abordaje transeptal ampliado. Los hallazgos intraoperatorios describieron una masa gelatinosa, de aspecto mucoídeo y color blanco amarillento, que se proyectaba desde la válvula mitral hacia la aurícula izquierda, de dimensiones aproximadas $40 \times 30 \mathrm{~mm}$, implantada en el anillo mitral posterior, inmediatamente inferior a los segmentos $\mathrm{P} 2$ y $\mathrm{P} 3$ del velo posterior mitral, en contacto con el aparato subvalvular y el músculo papilar posterior, pero sin invasión de dichas estructuras. Los velos de la válvula mitral tenían un aspecto normal, no adheridos al tumor. La porción móvil del tumor se proyectaba hacia la cavidad ventricular izquierda protruyendo hacia el TSVI (Figuras 3 y 4). La anatomía patológica confirmó, mediante microscopia e inmunohistoquímica con reacción positiva para calretinina, un tumor de matriz mixoide, compuesto por células de citoplasma basófilo, 


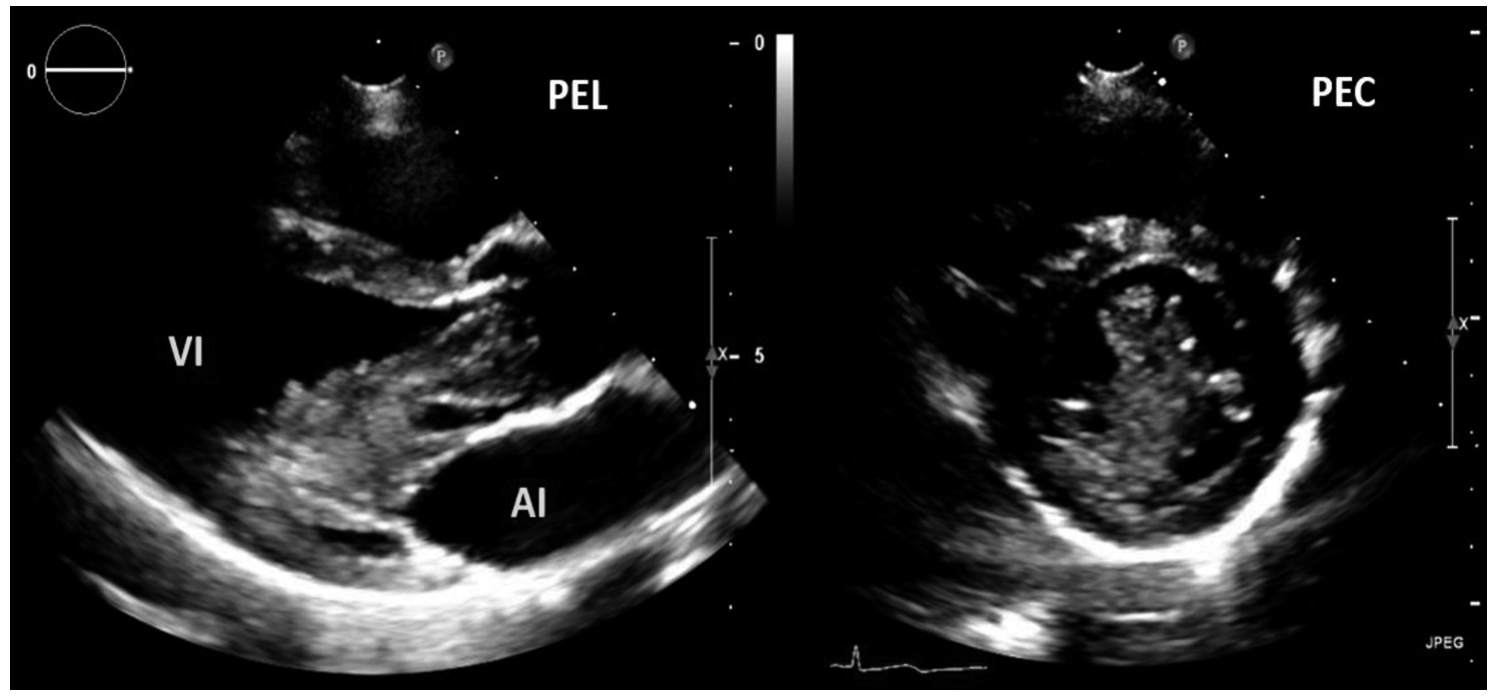

Figura 1. Ecocardiografía transtorácica (ETT). Panel izquierdo: Eje paraesternal largo (PEL) se observa tumor protruyendo hacia el TSVI en sístole. Panel derecho: Eje paraesternal corto (PEC) tumor adherido al anillo mitral posterior. VI: Ventrículo izquierdo. Al: Aurícula izquierda. Video y material complementario disponible en http://www.ecocardio.cl/mixoma.html

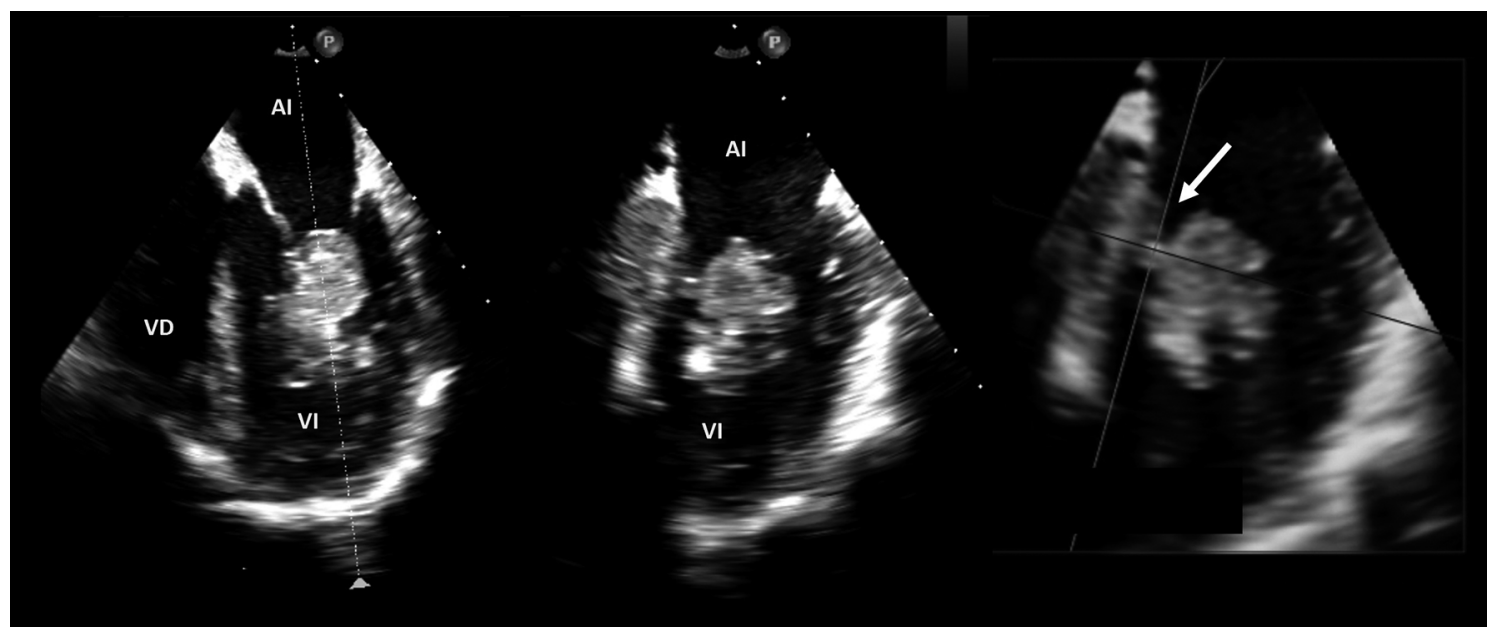

Figura 2. Ecocardiografía transesofágica (ETE). Panel izquierdo: ETE visón x-plane en $0^{\circ}$ y $90^{\circ}$ que muestra tumor adherido a anillo mitral. Panel derecho: Procesado de volumen 3D en QLAB que muestra punto de inserción (tallo) en anillo posterior de válvula mitral (flecha amarilla). Al: Aurícula izquierda. VI: Ventrículo izquierdo. VD: Ventrículo derecho. Video y material complementario disponible en http://www.ecocardio.cl/mixoma.html

fusiformes y de núcleos elongados e hipercromáticos compatible con un mixoma (Figura 5). La ecocardiografía de control intrapabellón y previo al alta, demostró la resección del mixoma y una insuficiencia mitral leve ya descrita en el estudio inicial. La paciente fue dada de alta al quinto día postoperatorio en buenas condiciones.

\section{Discusión}

Los tumores primarios del corazón son infrecuentes, siendo tres cuartas partes de ellos benignos. De este grupo, la mitad son mixomas cardíacos que tienen un aspecto macroscópico gelatinoso y mamelonado ${ }^{1}$. Estos tumores pueden 


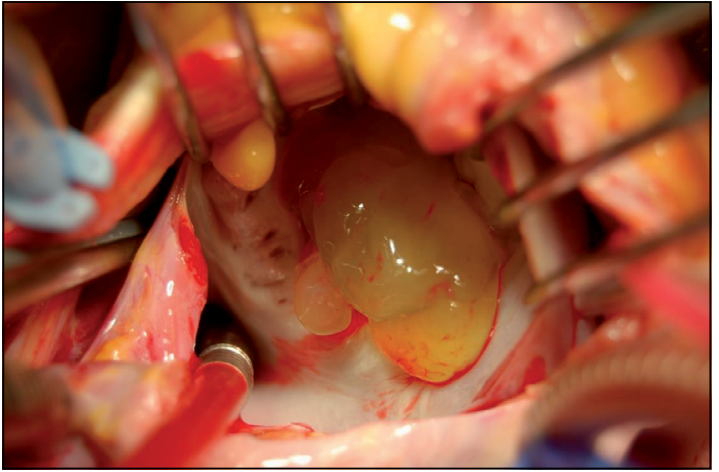

Figura 3. Hallazgo intraoperatorio. Fotografía intraoperatoria exponiendo el tumor desde un acceso transeptal ampliado.

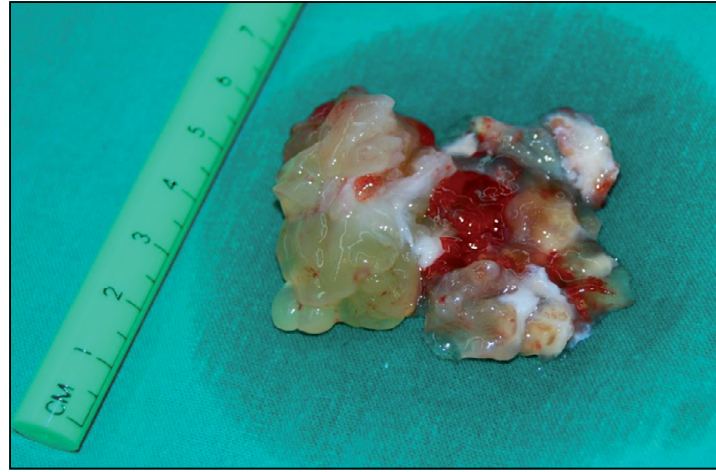

Figura 4. Tumor resecado. Fotografía de pieza operatoria.

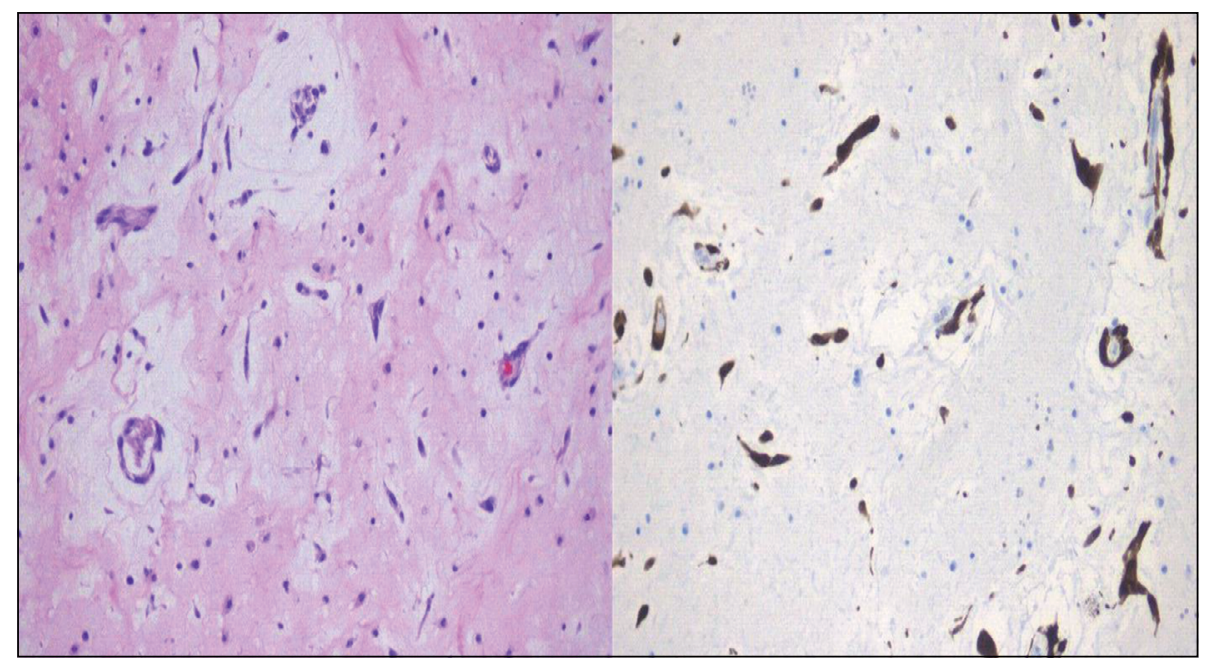

Figura 5. Anatomía patológica. Panel izquierdo: Tinción de hematoxilina-eosina, imagen microscopia. Panel derecho: Imagen de inmunohistoquímica con reacción positiva para Calretinina. presentarse de forma esporádica aislada o de forma sindromática con agregación familiar como en el complejo de Carney. En cuanto a su localización, lo más frecuente es la aurícula izquierda, en $75 \%$ de los casos, donde se encuentra habitualmente adherido por un tallo a la zona de la fosa oval. En segundo lugar se ubican en la aurícula derecha, en $15-20 \%$ de los casos $^{2}$. Sin embargo, existen otras localizaciones muy infrecuentes y de mayor riesgo, como la intraventricular izquierda (3-4\% de los casos $)^{1,3}$, que puede estar asociada a obstrucción al TSVI y muerte súbita ${ }^{4}$ y la relacionada a la válvula mitral. Esta última es de excepcional presentación, con aproximadamente 60 casos reportados en la literatura occidental ${ }^{5,6,7,8}$ y sin casos reportados en la literatura nacional, según nuestros registros?.

Respecto a su presentación clínica, los mixomas pueden manifestarse como un síncope o pre-síncope de esfuerzo en alrededor de $20 \%$ de los $\operatorname{casos}^{10,11}$, siendo un síntoma ominoso, ya que puede preceder a una muerte súbita. Con respecto al síncope de esfuerzo, es útil diferenciar si ocurrió durante el esfuerzo (sugiere cardiopatía estructural o arrítmico) o luego del esfuerzo (sugiere síncope mediado por reflejo). Sin embargo, independiente de esto debe ser considerado un síncope de alto riesgo y su evaluación de primera línea incluye una historia clínica completa, un examen físico exhaustivo con maniobras de provocación ortos- 
tática y un electrocardiograma de reposo ${ }^{12}$. Como segunda línea, y según cada caso, se prosigue con una ETT y un test de esfuerzo; sin embargo, si existe sospecha de cardiopatía estructural (miocardiopatía hipertrófica, lesiones valvulares, obstrucción al tracto de salida, tumores cardíacos) y el síncope ocurrió durante el esfuerzo, es prudente realizar la ETT previo al test de esfuerzo ${ }^{13,14}$.

En cuanto a los mixomas mitrales, estos agrupan los tumores que se originan en los velos mitrales, anillo, comisuras y aparato subvalvular (cuerdas y músculos papilares) y tienen algunas características distintivas ${ }^{6}$; se presentan mayormente en mujeres, tienen una menor tasa de síntomas constitucionales, son de menor tamaño ${ }^{15} \mathrm{y}$ se han asociado a alto riesgo de embolia y mayor riesgo de recurrencia. La principal serie reportada sobre dichos tumores describe su localización en: velo anterior $(43 \%)$, anillo mitral (7\%), velo anterior y anillo $(4,5 \%)$ y luego aparato sub-valvular con menor frecuencia ${ }^{6}$. En cuanto a sus principales complicaciones, están reportadas: insuficiencia mitral (33\%), obstrucción al TSVI $(33 \%)$ y estenosis mitral (7\%).

En el diagnóstico diferencial del mixoma deben considerarse los trombos y el fibroelastoma papilar, la RM de corazón con gadolinio otorga la posibilidad de realizar la caracterización tisular de las masas cardíacas y permite diferenciar con precisión entre pseudo-tumores (ej: trombos), tumores benignos y malignos ${ }^{16}$.

El tratamiento óptimo es la resección quirúrgica con la completa preservación valvular mitral, resultado poco frecuente de lograr (3\% de los casos sometidos a cirugía), como es el caso reportado. En 20\% de los pacientes es necesario el implante de una prótesis valvular mitral y, en $21 \%$, alguna técnica de reparación valvular6.

El caso que reportamos destaca en cuanto a su diagnóstico por su localización atípica (anillo posterior mitral) y por su gran tamaño, en contraposición a lo reportado históricamente ${ }^{15}$, y también en lo relativo a su resección, por lograr la preservación anatómica y funcional de la válvula mitral.

\section{Referencias}

1. Reynen K. Cardiac Myxomas. N Engl J Med 1995; 333: 1610-7.
2. Percell RL, Henning RJ, Siddique Patel M. Atrial myxoma: case report and a review of the literature. Heart Dis 2003; 5 (3): 224-30.

3. Díaz R, Aránguiz E. [Asymptomatic left ventricular myxoma incidentally diagnosed by echocardiography. Report of one case]. Rev Med Chile 2013; 141: 1594-7.

4. Prifti E, Ademaj F, Kajo E, Baboci A. A giant myxoma originating from the aortic valve causing severe left ventricular tract obstruction: a case report and literature review. World J Surg Oncol 2015; 13: 151.

5. Chakfé, N, Kretz, J G, Valentin P, Geny B, Petit H, Popescu S, et al. Clinical presentation and treatment options for mitral valve myxoma. Ann Thorac Surg 1997; 64 (3): 872-7.

6. Yuan S-M. Mitral valve myxoma: clinical features, current diagnostic approaches, and surgical management. Cardiol J 2012; 19 (1): 105-9.

7. Sandrasagra FA, Oliver WA, English TA. Myxoma of the mitral valve. Br Heart J 1979; 42 (2): 221-3.

8. Rocha ASC, Ferreira ME, Dutra PR, Rocha N, Tinoco $\mathrm{SM}$, Nascimento C, et al. Myxoma of the mitral valve. Arq Bras Cardiol 1999; 72 (5): 624-6.

9. Becker RP, Ramírez MA, Zalaquett SR, Morán S, Irarrázaval MJ, Arretz C, et al. Mixoma cardiaco: Caracterización clínica, métodos diagnósticos y resultados alejados del tratamiento quirúrgico durante tres décadas de experiencia. Rev Med Chile 2008; 136: 287-95.

10. Modi K, Venkatesh P, Agnani S, Rowland T, Reddy $\mathrm{P}$. Sudden death in a patient with left atrial myxoma: Report of two cases and review of literature. Br J Med Pract 2010; 3 (2): 48-51.

11. Kapoor MC, Singh S, Sharma S. Resuscitation of a patient with giant left atrial myxoma after cardiac arrest. J Cardiothorac Vasc Anesth 2004; 18 (6): 769-71.

12. Moya A, Sutton R, Ammirati F, Blanc J-J, Brignole M, Dahm JB. Guidelines for the diagnosis and management of syncope (version 2009). Eur Heart J 2009; 30 (21): 2631-71.

13. Lawlwess. CE. Syncope/Presyncope in the competitive athlete. En Lawless CE. Editor, Sports Cardiology Essentials: Evaluation, Management and Case Studies. Chicago, U.S.A. Springer 2011. p.163-73.

14. O'Connor FG, Oriscello RG, Levine BD. Exercise-related syncope in the young athlete: reassurance, restriction or referral? Am Fam Physician 1999; 60 (7): 2001-8.

15. Jaleski TC. Myxoma of the heart valves. Am J Pathol 1934; 10 (3): 399-406.

16. Sparrow PJ, Kurian JB, Jones TR, Sivananthan MU. MR imaging of cardiac tumors. Radiographics 2005; 25: 1255-76. 